\title{
TTG2-regulated development is related to expression of putative AUXIN RESPONSE FACTOR genes in tobacco
}

Qian Zhu ${ }^{1+}$, Baoyan $\mathrm{Li}^{1,2+}$, Shuyuan Mu${ }^{1}$, Bing Han ${ }^{1}$, Runzhi Cui ${ }^{1}$, Manyu Xu', Zhenzhen You ${ }^{1}$ and Hansong Dong ${ }^{1 *}$

\begin{abstract}
Background: The phytohormone auxin mediates a stunning array of plant development through the functions of AUXIN RESPONSE FACTORS (ARFs), which belong to transcription factors and are present as a protein family comprising 10-43 members so far identified in different plant species. Plant development is also subject to regulation by TRANSPARENT TESTA GLABRA (TTG) proteins, such as NtTTG2 that we recently characterized in tobacco Nicotiana tabacum. To find the functional linkage between TTG and auxin in the regulation of plant development, we performed de novo assembly of the tobacco transcriptome to identify candidates of NtTTG2-regulated ARF genes.
\end{abstract}

Results: The role of NtTTG2 in tobacco growth and development was studied by analyzing the biological effects of gene silencing and overexpression. The NtTTG2 gene silencing causes repressive effects on vegetative growth, floral anthocyanin synthesis, flower colorization, and seed production. By contrast, the plant growth and development processes are promoted by NtTTG2 overexpression. The growth/developmental function of NtTTG2 associates with differential expression of putative ARF genes identified by de novo assembly of the tobacco transcriptome. The transcriptome contains a total of 54,906 unigenes, including 30,124 unigenes (54.86\%) with annotated functions and at least 8,024 unigenes (14.61\%) assigned to plant growth and development. The transcriptome also contains 455 unigenes (0.83\%) related to auxin responses, including 40 putative ARF genes. Based on quantitative analyses, the expression of the putative genes is either promoted or inhibited by NtTTG2.

Conclusions: The biological effects of the NtTTG2 gene silencing and overexpression suggest that NtTTG2 is an essential regulator of growth and development in tobacco. The effects of the altered NtTTG2 expression on expression levels of putative ARF genes identified in the transcriptome suggest that NtTTG2 functions in relation to ARF transcription factors.

\section{Background}

TRANSPARENT TESTA GLABRA (TTG) proteins were identified as regulators of trichome and seed development in plants [1-4]. TTGs are characterized by the presence of the WD40 motif [5-7], which refers to the conserved tryptophan (W) and aspartate (D) dipeptide and the length of approximately 40 amino acid residues in a protein sequence [8]. This motif is present as a series of repeats to form protein interaction domain in a variety of proteins [9-11], including all TTGs so far

\footnotetext{
* Correspondence: hsdong@njau.edu.cn

${ }^{\dagger}$ Equal contributors

'Plant Growth and Defense Signaling Laboratory, State Ministry of Education Key Laboratory of Integrated Management of Crop Pathogens and Insect Pests, Nanjing Agricultural University, Nanjing 210095, China Full list of author information is available at the end of the article
}

identified except for Arabidopsis thaliana AtTTG2, which is a WRKY transcription factor [12]. As the WD40 domain has multiple modes for recruiting different substrates, WD40-containing proteins can serve as interchangeable substrate receptors and interact with diverse types of proteins $[11,13]$. Due to this characteristic, TTGs have the potential of regulating both development and defenses in plants $[5,7,14,15]$.

Depending on natures of interacted proteins, TTGs differentiate their functions between development and defense regulations [6,16]. In Arabidopsis, AtTTG1 interacts with the bHLH transcription factor GL3 while recruiting MYB GL1, forming the WD40-bHLH-MYB complex that acts to regulate trichome development $[13,17]$. Previously we demonstrated that WD40 enabled

\section{Biomed Central}


tobacco (Nicotiana tabacum) TTG1 (NtTTG1) to interact with ParA1 [7], an elicitin protein produced by an oomycete pathogen and inducing hypersensitive cell death in the plant [18]. The NtTTG1-ParA1 interaction was essential for the induction of cell death first in leaf trichomes and then in mesophyll cells [7]. NtTTG2 is an analog of NtTTG1 and both proteins share four WD40 repeats $[5,7,19]$. Recently we showed that NtTTG2 suppressed tobacco resistance to pathogens by indirectly modulating subcellular localization of the NPR1 protein [5], which is a transcriptional regulator of defense responses to pathogens [20-22]. NtTTG2 does not interact with NPR1 but is able to sequester NPR1 from the nucleus, thus preventing NPR1 from transcriptional regulation of defense responses [5]. These studies suggest that TTGs regulate specific processes of development or defenses by either directly or indirectly interacting with other proteins involved in the processes. However, whether NtTTG2 plays a role in development regulation was unclear.

Plant development is subject to a complex regulation of phytohormones with auxin playing a significant role. Auxin mediates a stunning array of the development $[23,24]$ through the functions of AUXIN RESPONSE FACTOR (ARF) proteins in transcriptional regulation of auxin responses [25-31]. ARF transcription factors constitute a phylogenic protein family [25,29-32] made of 10-43 members as identified so far in different plant species [33-36]. It is accepted that different members of the ARF protein family target distinct types of auxin-responsive genes, such as those classified in the Small Auxin Up RNA (SAUR), Auxin/Indole-Acetic Acid inducible (Aux/IAA), and Gretchen Hagen 3 (GH3) gene families $[26,28,31,32,37-40]$. However, only a few of auxinresponsive genes have been shown definitely as ARF targets and to be regulated directly by specific ARFs [28,31]. In particular, little was known about the regulation of auxin responses in tobacco. In the plant, only four auxin-responsive genes were identified, whereas, at least 29, 134, and 15 members of Aux/IAA [23], SAUR [41], and $G H 3$ [42] gene families were characterized in other plants. In tobacco, moreover, only ARF1 [43] and ARF8 (JN835423.1) were cloned with full length cDNA sequences while additional eleven $A R F$ homologs were identified as expressed sequence tags (ESTs) [44] in contrast to the 10-43 members of the ARF protein family already identified in other plants [33-36]. Therefore, it is rational to conjecture that tobacco may possess more $A R F \mathrm{~s}$ and auxin-responsive genes than the currently known numbers. In addition, there is as yet no study to characterize functions of ARFs in tobacco.

This study was attempted to elucidate the role of NtTTG2 in the growth and development of tobacco and to identify $A R F$ gene candidates associated with NtTTG2- regulated growth and development. We show that NtTTG2 is required for the vegetative growth and the development of flowers and seeds. We performed de novo assembly of the tobacco $N$. tabacum transcriptome and analyzed functional components in the transcriptome. By using transcriptome information, we identified the unigenes assigned to auxin responses and representing putative $A R F$ genes in particular. We present evidence that NtTTG2 affects the expression of putative $A R F$ genes in the plant.

\section{Results}

The repressive effect of NtTTG2 silencing on the vegetative growth and the development of flowers and seeds

\section{in tobacco}

To elucidate the roles of NtTTG2 in the growth and development of tobacco $N$. tabacum, we generated the NtTTG2-silencing line TTG2RNAi4 under background of the N. tabacum variety $\mathrm{NC} 89$ and observed its growth and development in contrast to the wild-type (WT) phenotype. The transgenic NC89 line WT RFP was used as a control since it highly resembled $W T$ in the development process (Additional file 1: Figure S1a) and biological productivity (Additional file 1: Figure S1b,c). RFP is a red-fluorescent protein-encoding gene used in detecting subcellular localization of the protein [5] but is not related to this study. Molecular and genetic characterizations of transgenic plants have been documented in a recently published article [5].

In this study, we investigated the vegetative growth of WT RFP and TTG2RNAi4 plants on Murashige and Skoog (MS) agar medium and in pots. We also analyzed the expression of the NtTTG2 gene along with the expansin (EXP) protein-encoding genes EXP1 and EXP2, which are responsive to auxin and contribute to plant growth $[45,46]$. When plants were grown on the medium, the root length of TTG2RNAi4 was much shorter than that of WT RFP (Figure 1a), and this difference was statistically significant $(P<0.05$ or 0.01$)$ as scored in $5-20$ days (Figure 1b). Similar results were observed on plants grown in pots (Figure 1c), and compared to WT RFP, TTG2RNAi4 was significantly $(P<0.05$ or 0.01$)$ impaired in the vegetative growth based on the biomass scored as height (Figure 1d) and weight (Figure 1e) of fresh plants. Consistently, the expression of NtTTG2, EXP1, and EXP2 genes was detected at substantial levels in both roots (Figure 1f) and leaves (Figure 1g) of WT RFP, but little in the TTG2RNAi4 plant. These analyses indicate that NtTTG2 is required for the vegetative growth of tobacco in correlation with the expression of EXP1 and EXP2 in the plant.

Then, we observed the flower and seed development, which has been shown to be regulated by TTG proteins in Arabidopsis [16] and petunia [2,4]. We analyzed the effects of NtTTG2 silencing on the floral development 


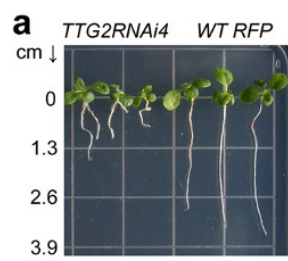

C WT RFP TTG2RNAI4
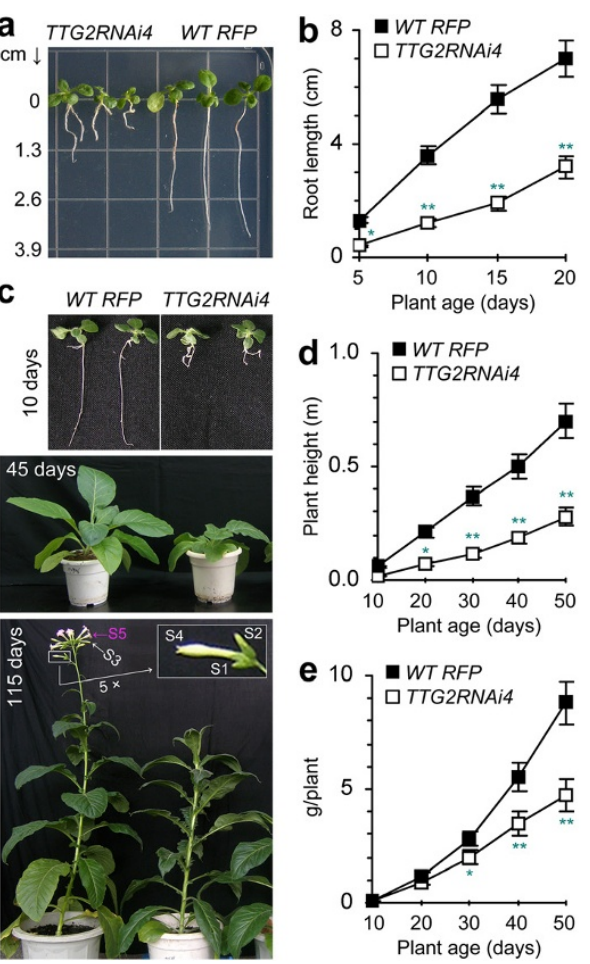

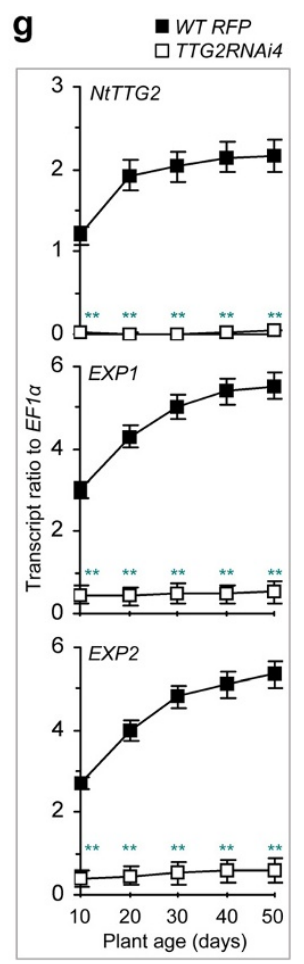

Figure 1 The effect of NtTTG2 silencing on tobacco growth. The NtTTG2-silenced tobacco line TTG2RNAi4 was compared to the control WT RFP plant in terms of the vegetative growth and the expression of NtTTG2 and EXP genes. (a, b) Root monitoring after 10-day growth on the medium. (c) Plants grown in pots. Floral development stages S1-S5 are indicated. (d, e) The biomass of plants from (c). (f) Real-time RT-PCR analyses of the indicated genes expressed in roots of plants from (b). (g) Real-time RT-PCR analyses of gene expression in leaves of 45-day-old plants grown in pots and equivalent to those in (c). In ( $\mathbf{b}, \mathbf{d}-\mathbf{g})$, data shown are mean values \pm standard deviation (SD) bas of results from five experimental repeats (50 plants/repeat). Data were analyzed by the ANOVA method along with one-way Fisher's Least Significant Difference (LSD) test. Significant differences between data pairs at the corresponding time points are indicated by single asterisks $(P<0.05)$ or double asterisks $(P<0.01)$ on the graphs.

throughout stages S1-S5 (Figures 1c and 2a). Average time to $\mathrm{S} 1$ was 105 and 143 , and average time from S1 to S5 was 7 and 9 days in WT RFP and TTG2RNAi4 plants, respectively. The effect of NtTTG2 silencing on flower color was marked. Plants produce anthocyanin, a water-soluble pigment that may make flowers differently colored [47]. WT RFP flowers had visible anthocyanin production since S4 and petals appeared red at S5 (Figure 2a). Floral anthocyanin extracts (Figure 2b) and spectrophotometric analyses (Figure 2c) indicated that WT RFP flowers started to produce anthocyanin at S3 and that abundant amounts of the pigment were detected at S4 and S5. By contrast, little anthocyanin was found in TTG2RNAi4 flowers (Figure 2a-c).

Next, we correlated the variation of anthocyanin production with the expression of the DFR and ANS genes involved in late stage of the anthocyanin biosynthesis pathway that mainly comprises nine genes [48-50]. The floral expression of DFR and ANS was NtTTG2dependent (Figure 2d) but that of the other genes was not (Additional file 2: Figure S2). In WT RFP, the expression of NtTTG2, DFR, and ANS was increased with the floral development, but the three genes were little expressed in TTG2RNiAi4 (Figure 2d). Therefore, NtTTG2 was required for $D F R$ and $A N S$ expression with consequent effects on anthocyanin production and flower colorization (Figure 2a-c). In addition, NtTTG2 was also required for seed production as NtTTG2 silencing caused acute seed abortion (Figure 2e), and more than 97\% seeds were aborted in TTG2RNAi4 compared to WT RFP (Figure 2f). These analyses indicate that NtTTG2 functions in flower and seed development of tobacco.

\section{The promoting effect of NtTTG2 overexpression on tobacco growth and development}

To further demonstrate the role of NtTTG2 in tobacco growth and development, we generated the NtTTG2overexpressing (P35S:TTG2:RFP) NC89 lines [5] and observed their growth and development characters in comparison with the WT RFP plant. Here, P35S refers to the cauliflower mosaic virus $35 \mathrm{~S}$ promoter. In all tested characters, P35S:TTG2:RFP lines were opposite to TTG2RNiAi4. Compared to WT RFP, P35S:TTG2:RFP plants grew better (Figure 3a) and had higher levels of EXP expression (Figure 3b) and biomass (Figure 3c). P35S: TTG2:RFP plants also had better flower colorization 

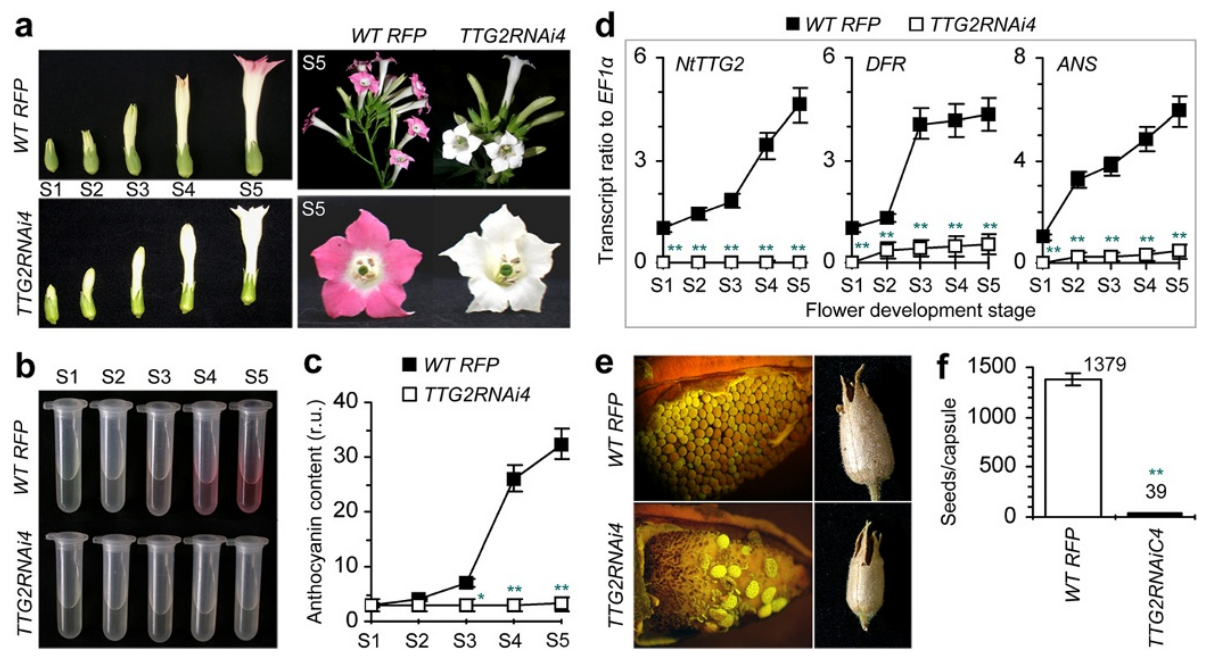

Figure 2 The effects of NtTTG2 silencing on flower and seed development. (a) Flowers at five development stages (S1-S5) from 120-d-old WT/RFP and 160-d-old TTG2RNAi4 plants. (b, c) Flower anthocyanin extracts and spectrophotometric analyses. The floral anthocyanin content was quantified as relative unit (r.u.). (d) Real-time RT-PCR analyses of floral transcripts of the NtTTG2 gene as well as the DFR and ANS genes, which are involved in the late stage of the anthocyanin biosynthesis pathway. (e) Growing fruits (left) from 140-d-old WT/RFP and 170-d-old TTG2RNAi4, and mature fruits (right) from 170-d-old WT/RFP and 210-d-old TTG2RNAi4 plants. (f) The number of seeds per mature fruit. Data were given as mean values \pm SD bars of results from three $(\mathbf{c}, \mathbf{d})$ and five (f) experimental repeats (30 fruits/repeat). Significant levels $\left({ }^{*} P<0.05 ;{ }^{* *} P<0.01\right)$ in differences between corresponding data pairs were analyzed by one-way ANOVA and LSD test.

(Figure 3d), higher levels of NtTTG2, DFR, or ANS expression from S1 through S5 (Additional file 2: Figure S2), and more anthocyanin production in $\mathrm{S} 4$ flowers (Figure 3e). Moreover, P35S:TTG2:RFP capsules were well developed (Figure 3d) with more seeds per fruit (Figure 3f). Significant $(P<0.01)$ differences were found between WT RFP and each line of P35S:TTG2:RFP while P35S:TTG2:RFP\#1 was the best in these developmental characters. Clearly, NtTTG2 overexpression causes a promoting effect on the vegetative growth and the development of flowers and seeds in tobacco.

\section{Transcriptome sequencing and de novo assembly}

We sought to identify $A R F$ gene candidates that might associate with NtTTG2-regulated growth and development in the tobacco $N$. tabacum variety NC89. We performed de novo assembly of the transcriptome by using the RNA-Seq technique and a mixture of equal quantities of total RNA isolated from leaves of WT RFP, TTG2RNAi4, and P35S:TTG2:RFP\#1. The mixed RNA sample from three biological repeats was used in the library construction and the transcriptome sequencing with the Illumina HiSeq 2000 sequencer, followed by de novo assembly with the Trinity assembler.

In all, 30,325,328 raw reads were generated, and a total of 29,573,259 clean reads, occupying $97.52 \%$ of total, were obtained after three types of reads (containing adaptor sequences, more than $10 \%$ unknown bases, and more than half of the quality values of the bases were lower than 5) were removed by Illumina RNA-Seq deep- sequencing (Figure 4a). Using the high quality reads, 111,207 transcripts were assembled with an average length of 1,031 bp, a length variation in $201-17,548 \mathrm{bp}$, and $15,824(14.23 \%)$ transcripts longer than $2,000 \mathrm{bp}$. Redundancy rate in 111,207 transcripts was $50.63 \%$, and the longest copy of redundant transcripts was regarded as a unigene [51,52]. In total, 54,906 unigenes were identified in the transcriptome and their length was found to vary from $201 \mathrm{bp}$ to $17,548 \mathrm{bp}$ (Figure $4 \mathrm{~b}$ ). The length of 33,116 (60.31\%), 9,727 (17.72\%), and 7,617 (13.87\%) unigenes ranges accordingly in 201-500, 501-1,000, 1,0012,000 bp while $4,446(8.10 \%)$ unigenes are longer than $2,000 \mathrm{bp}$.

\section{Functional annotation of unigenes}

Sequences of assembled unigenes were searched against seven different databases (Figure 5) with an E-value threshold of $10^{-5}$ (1.0E-5). As shown in Figure 5, various numbers of unigenes showed significant similarity to the lodged in those databases; similar sequences of 30,124 (54.86\%) unigenes were found in at least one database while 4,009 (7.3\%) unigenes shared similarity to sequences deposited in all databases. However, sequence orientation of 24,782 (45.14\%) unigenes is still unknown. Based on the E-value distribution of the top hits, $57.45 \%$ of the mapped unigene sequences showed significant homology (less than 1.0E-50) with genes deposited in the Nr (NCBI non-redundant protein sequences) database (Figure 6a). By searching against the same database, $86.69 \%$ and $40.82 \%$ of the unigenes were found to 


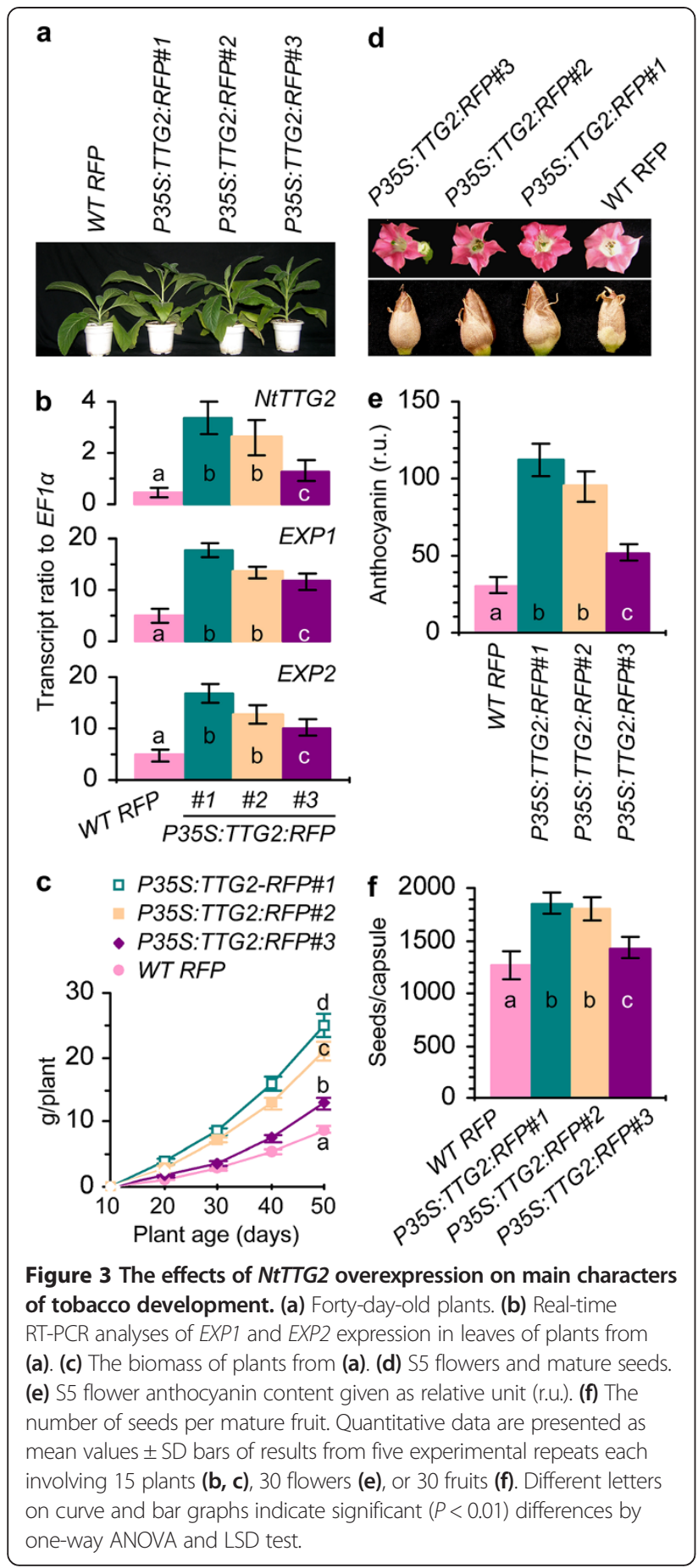

possess sequence similarities greater than $50 \%$ and $75 \%$, respectively (Figure $6 \mathrm{~b}$ ). In regard to organism sources of homologs, approximately $99.57 \%$ of unigenes with $\mathrm{Nr}$ annotations were matched to plant genes (Figure 6c). While $10.83 \%, 10.76 \%, 6.80 \%, 5.95 \%, 36.65 \%$ of the mapped unigene sequences possess significant similarity, respectively with orthologs of Ricinus communis, Populus trichocarpa, Nicotiana tabacum, Glycine max, and Vitis vinifera (Figure 6d).

\section{Functional classification of unigenes}

Functions of the unigenes were classified with GO (Gene Ontology), an international standardized gene functional classification system that defines genes in three ontologies: "molecular function", "cellular component", and "biological process". We used the Blast2GO program [53] to obtain $\mathrm{GO}$ annotation of the unigenes in the $\mathrm{Nr}$ database. Then, we used the WEGO software [54] to classify the unigenes to GO function terms. In total, 24,623 unigenes (44.85\%) have GO annotation, and these unigenes are assigned to 58 functional terms (Figure 7). Of these 24,623 unigenes, the "biological process" occupied the majority $(89,767,46.14 \%)$, followed by "cellular component" $(71,929,36.97 \%)$ and "molecular function" $(32,846,16.88 \%)$. Around the comprehensive range of GO categories, "cellular process" (16,726, 8.60\%), "cell" (15,739, 8.10\%), "cell part" (15,680, 8.06\%), "metabolic process" (15,460, 7.95\%), and "binding" (14,641, 7.53\%) proteins are in the top five rank and make up the majority of the unigenes (Figure 7). "Cell proliferation", "extracellular matrix part", and "nucleotide proteins" (not shown in Figure 7 due to small values) together occupy only $0.92 \%$ of unigenes that have GO annotation. In particular, numbers of unigenes are $614(2.49 \%$ of total number of GO-annotated genes), 2,943 (11.95\%), 2,277 (9.25\%), and 2,190 (8.89\%) assigned to "growth", "development process", "reproduction", "reproductive process" in the biological process category (Figure 7). Moreover, 3,248 (13.19\%) unigenes fall into the functional term of transcription factor activities in the "molecular function category" (Figure 7).

Functions of the unigenes were also classified with the KOG/COG (euKaryotic Ortholog Groups/Clusters of Orthologous Groups of Proteins) databases that classify predicted unigene products into distinct functional groups [55]. By the KOG/COG analysis, 10,152 of the unigenes were assigned to the 26 COG classifications (Figure 8). Some single unigenes were assigned to several COG categories, and this resulted in a record of 11,439 unigenes in all COG classifications. In comparison, the category of "General function prediction" contains the greatest number of unigenes $(1,874,16.38 \%)$, followed by "Post-translational modification, protein turnover, and chaperon" (1,346, 11.77\%), "Signal Transduction" (970, $8.48 \%$ ), "Intracellular trafficking and secretion" (692, $6.05 \%)$, "Transcription" (627, 4.48\%), and "RNA processing and modification" (539, 4.72\%).

\section{Metabolic pathway analysis of unigenes}

Orientation of unigenes in metabolic pathways was analyzed with the KEGG (Kyoto Encyclopedia of Genes and 


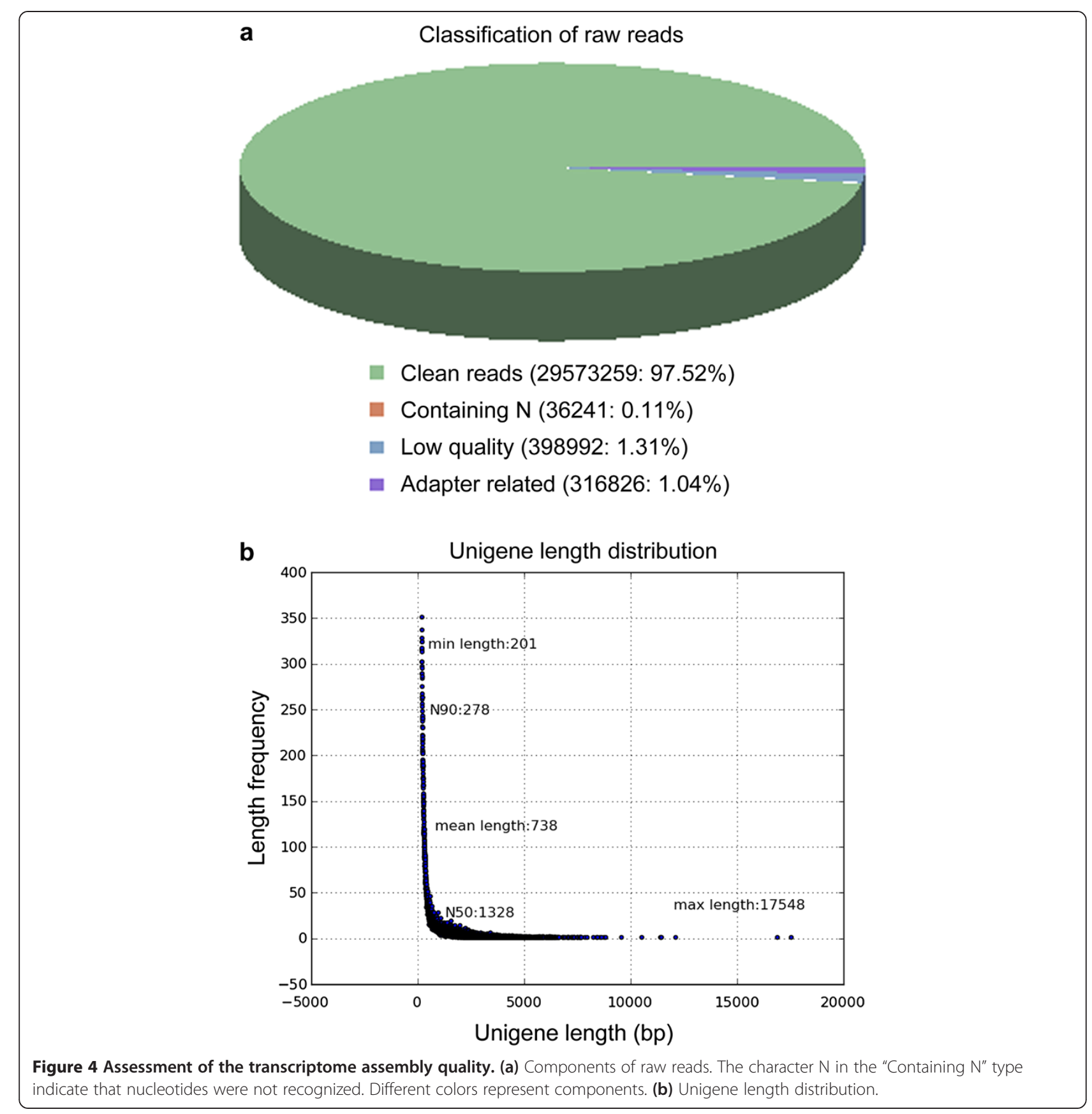

Genomes) database, which integrates genomic, biochemical, and functional information in various cellular processes [56]. Based on BLASTx searches with an E-value threshold of $10^{-5}, 7,529$ of the 54,906 unigenes were assigned to 31 KEGG pathways in the KEGG database (Figure 9). Those pathways were divided into five branches with numbers/percents of unigenes shown in parentheses: "Cellular Processes" (902/1.64\%), "Environmental Information Processing" (658/1.20\%), "Genetic Information Processing" (1,830/3.33\%), "Metabolism"
(3,255/5.93\%), and "Organismal Systems" (1,192/2.17\%). Of the 31 KEGG pathways, moreover, the pathway of "carbohydrate metabolism" contains homologs of the most unigenes (724/1.32\%), followed by "translation" (665/1.21\%), "folding, sorting and degradation" (625/ $1.14 \%)$, "signal transduction" (622/1.13\%), "energy metabolism" (506/0.92\%), and "amino acid metabolism" (469/0.85\%), whereas, only no more than 10 unigenes were assigned to "signaling molecules and interaction" and "sensory system". 


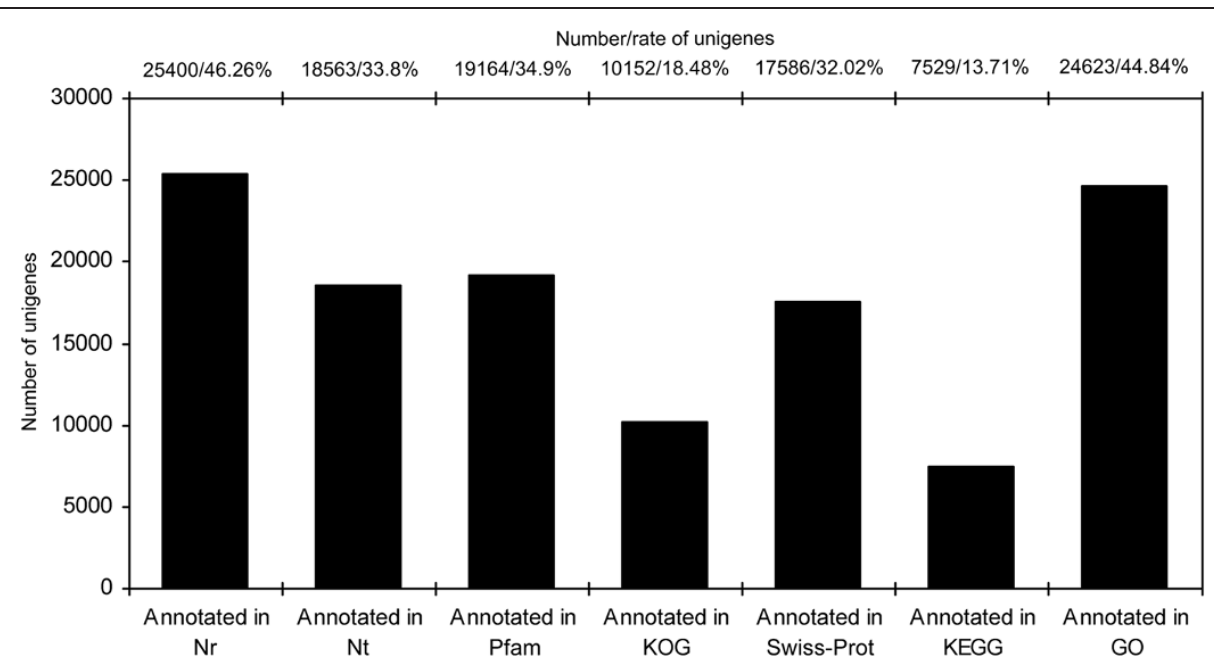

Figure 5 Distributions of unigenes in seven public databases.

Unigenes assigned to auxin responses and the effects of NtTTG2 on the expression of putative ARF genes

Browsing of the tobacco transcriptome profile identified 455 unigenes assigned to the regulation of auxin responses in plants (Figure 10a). In particular, 40, 39, 15, and 33 unigenes were predicted to be $A R F, A u x / I A A$,
GH3, and SAUR gene candidates while additional 10, 58, and 108 unigenes were categorized as auxin perception, auxin signaling pathway, and response to auxin stimulus, respectively. This suggests that the tobacco transcriptome is suited for a potential use in further studies to characterize the regulation of auxin responses in the
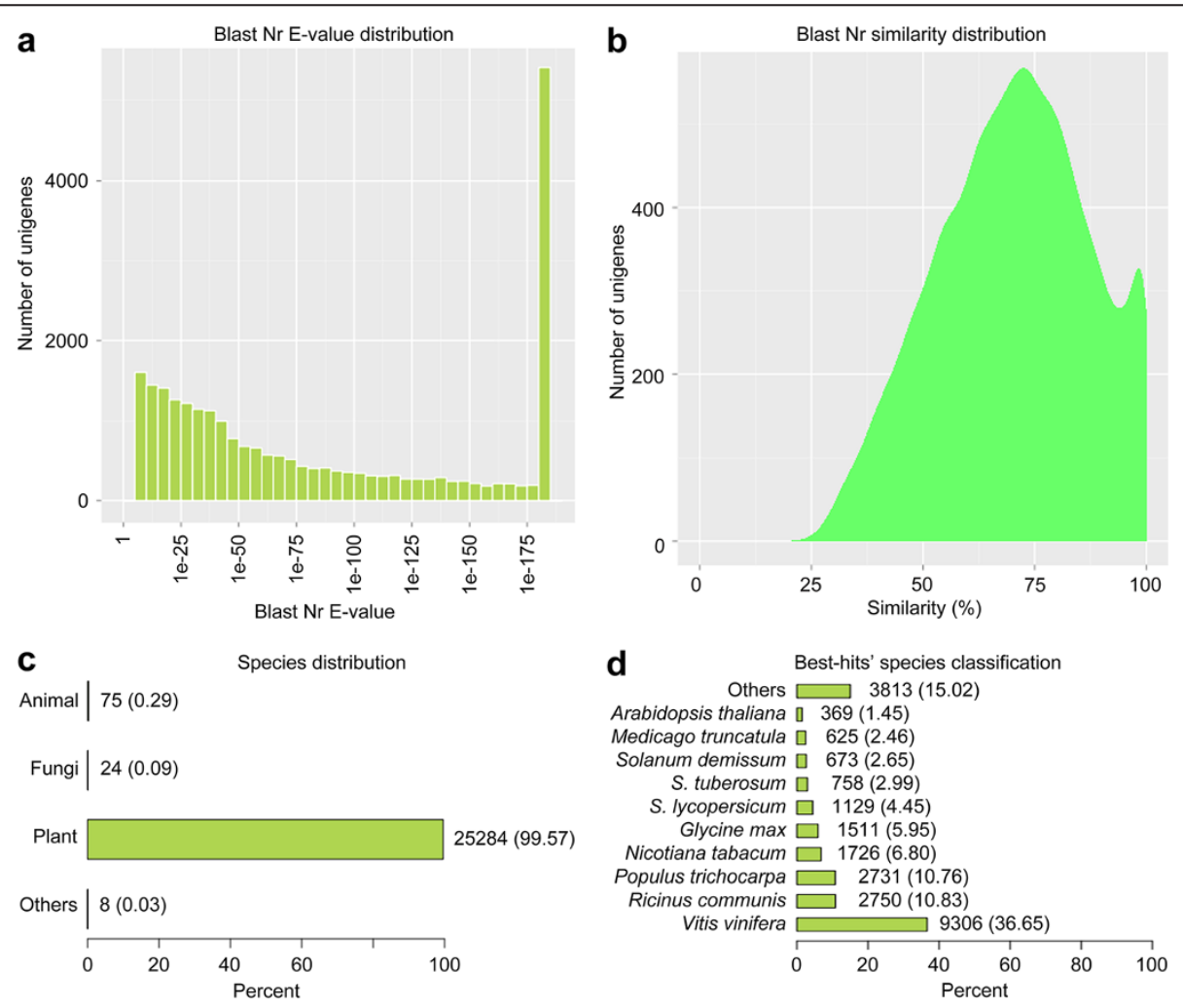

Figure $\mathbf{6}$ Characteristics of similarity search against $\mathrm{Nr}$ databases. (a) E-value distribution of BLAST hits for unigenes with a cutoff E-value of 1.0E-5. (b) Similarity distribution of the top BLAST hits for unigenes. (c, d) Organism species distribution of the top BLAST hits for unigenes in Nr database. The numbers of unigenes are indicated together with percentages placed in parentheses. 


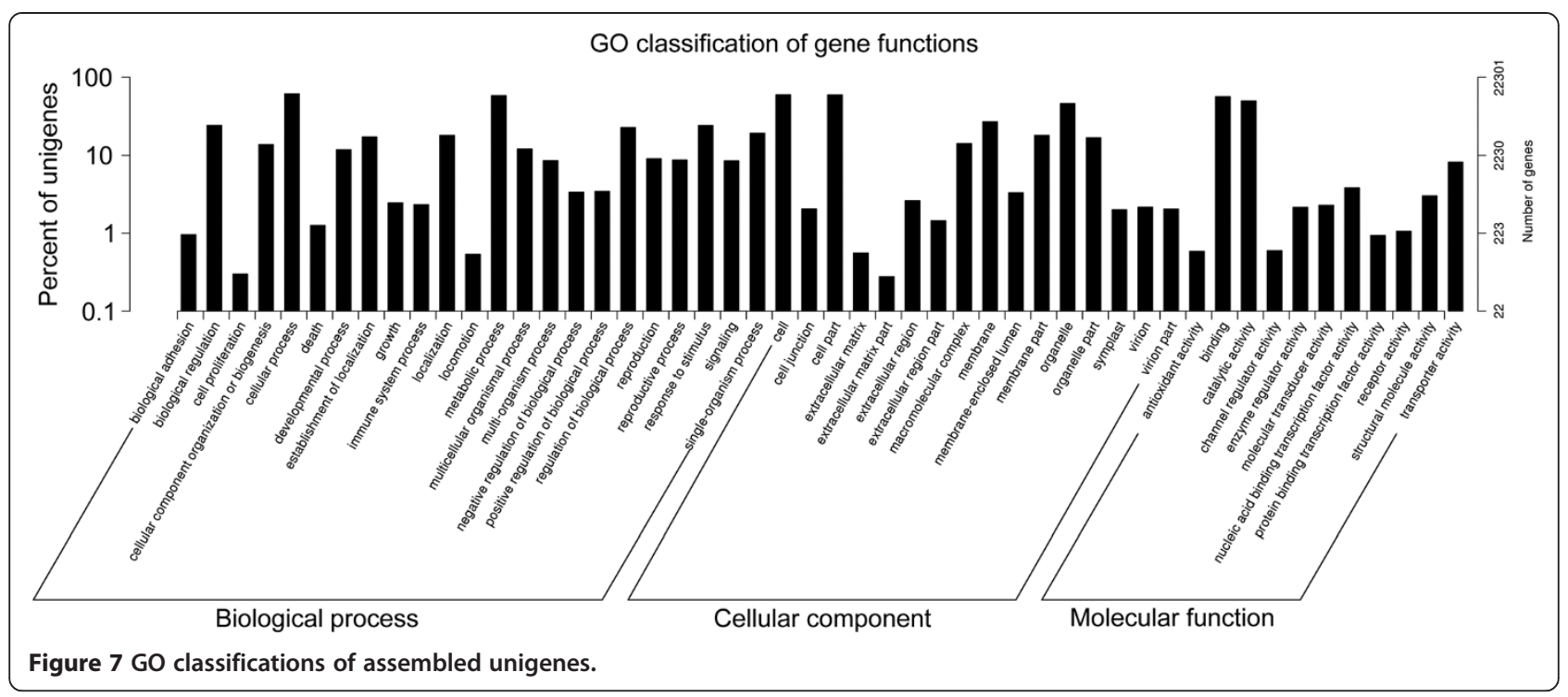

plant. To examine this notion, we analyzed the effects of NtTTG2 on the expression of the 40 putative ARF genes (Additional file 3: Table S1) by testing their expression in WT RFP, TTG2RNAi4, and P35S:TTG2:RFP\#1 plants. As shown in Figure 11, expression levels of 27 putative $A R F$ genes were similar but the other 13 candidates were expressed at different extents in the three genotypes of tobacco. Of the 13 differently expressed putative ARF genes, two (comp26539_c0 and comp40625_c0) were downregulated by NtTTG2 as their transcript abundances were decreased in P35S:TTG2:RFP\#1 but increased in TTG2RNAi4, compared to WT RFP used as a transgenic control plant. Inversely, the other 11 putative $A R F$ genes (comp1238_c0, comp19484_c2, comp30272_c0, comp 31328_c0, comp31531_c0, comp38086_c0, comp38146_ c2, comp39443_c0, comp41729_c1, comp41729_c2, and comp42904_c0) were upregulated by NtTTG2, and the expression of these putative genes was enhanced in P35S: TTG2:RFP\#1 but suppressed in TTG2RNAi4, compared to the control plant. Therefore, NtTTG2 indeed affects

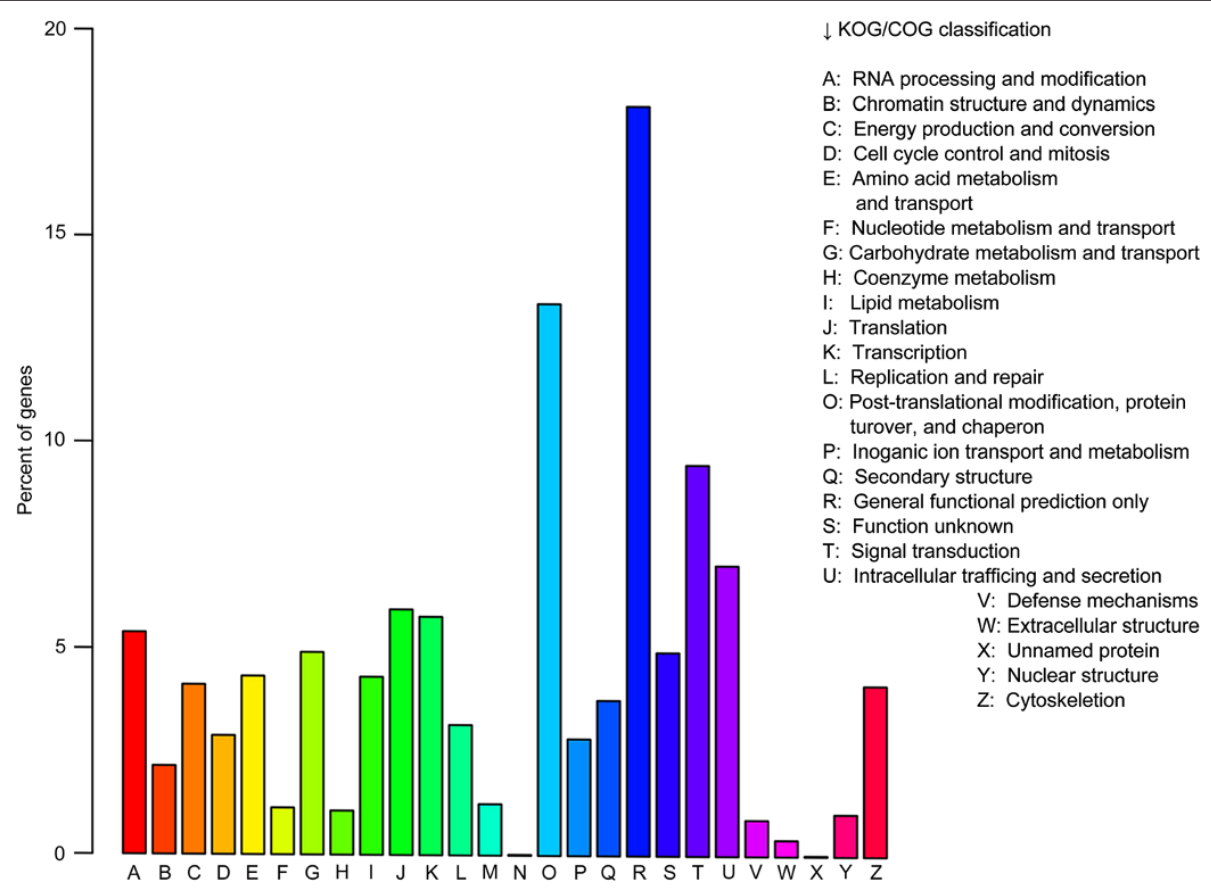

Figure $8 \mathrm{KOG} / \mathrm{COG}$ classification of unigenes. 

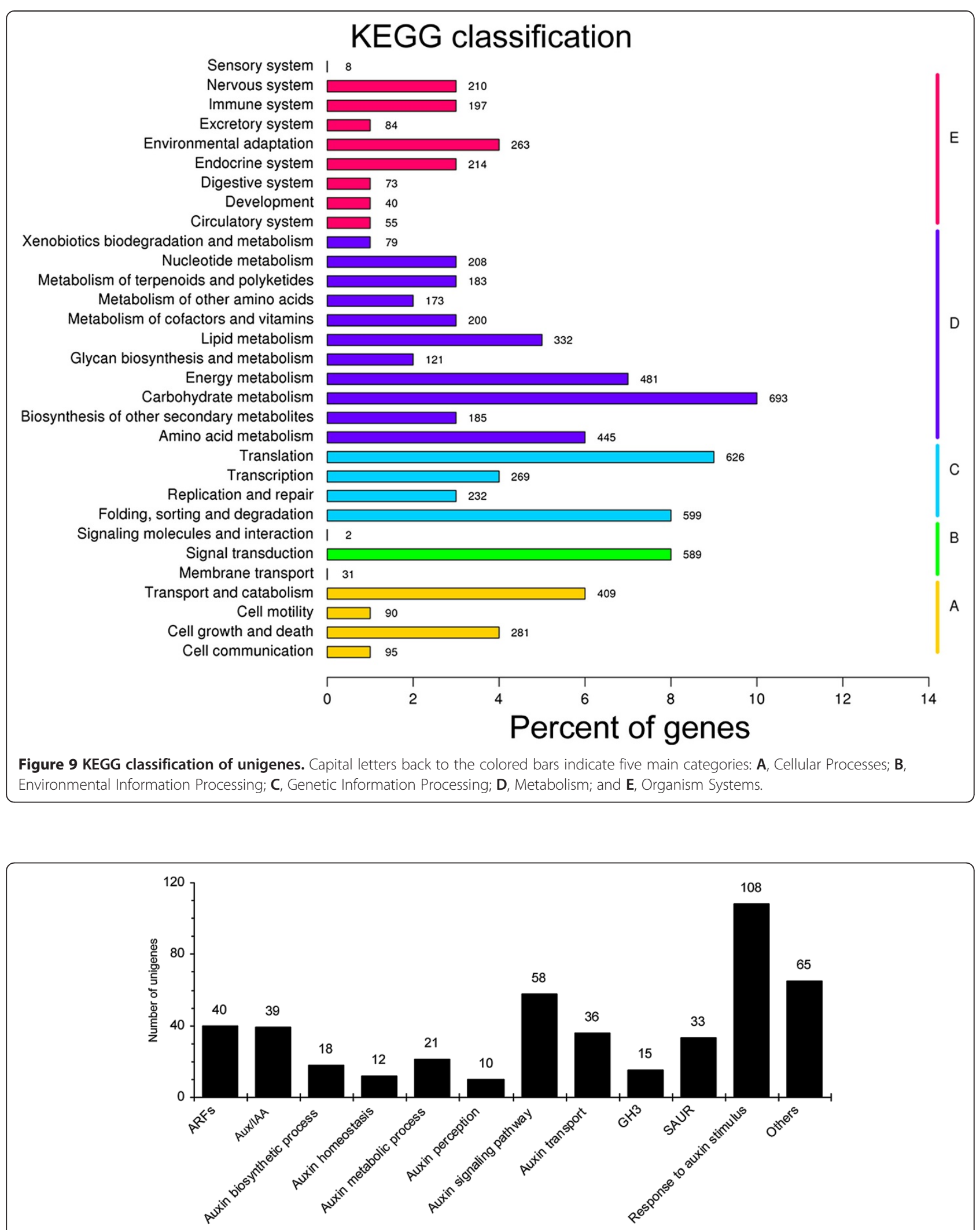

Figure 10 Phylogenic protein families involved in auxin responses and identified in the transcriptome. The number of genes in the corresponding protein family group (bottom character panel) is shown on top of the bar graph. The "Others" group mainly contains auxinrepressed and auxin-induced proteins that do not belong to any of the designated groups. 


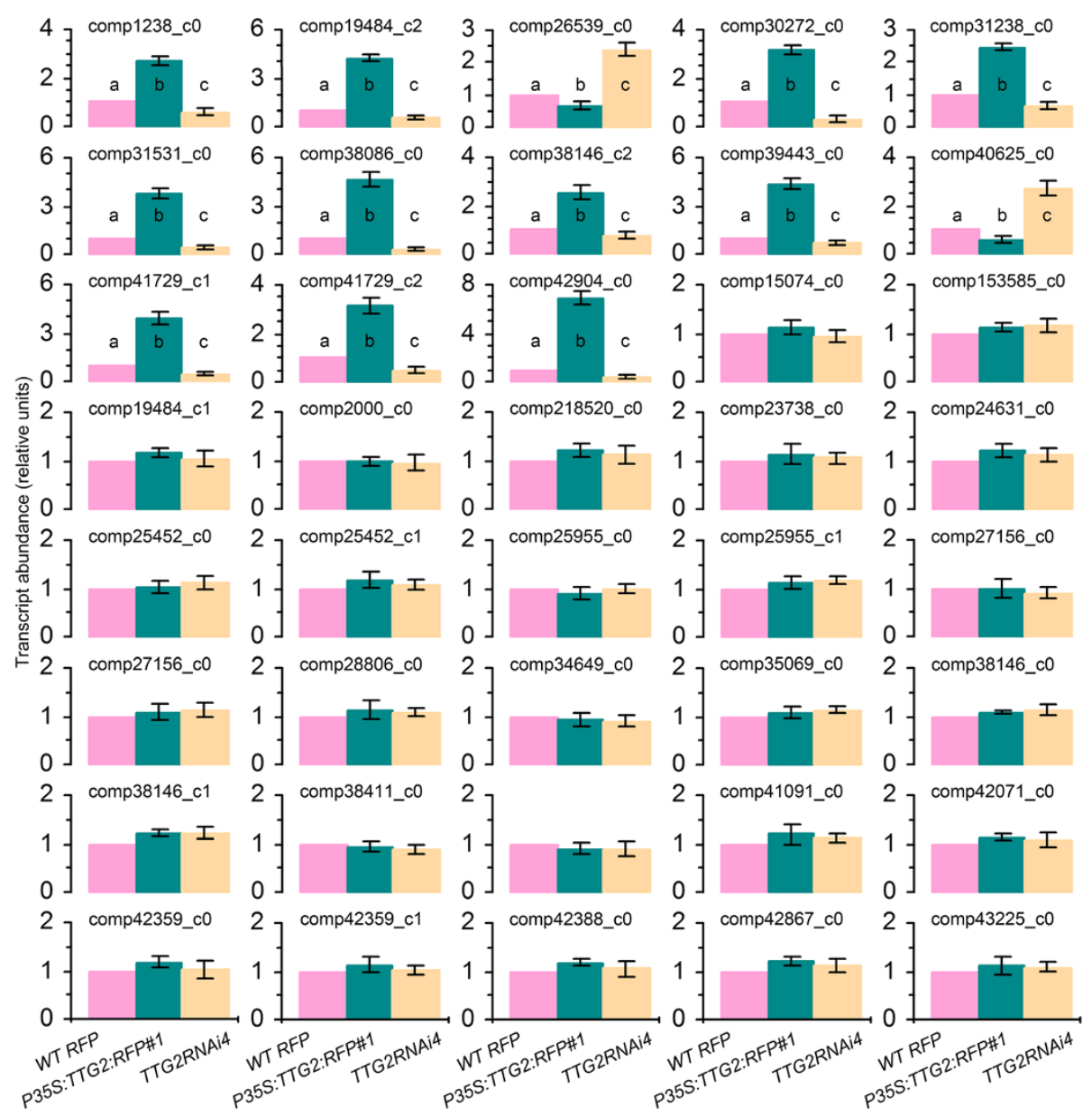

Figure 11 The effects of NtTTG2 on the expression of putative ARF genes. The effects were determined by analyzing the expression of putative ARF genes in WT RFP, P35S:TTG2:RFP\#1, and TTG2RNAi4 plants. Putative ARF genes are shown as identification code in the transcriptome inventory. Gene expression levels in the control plant were regarded as 1 to qualify relative amounts of the same gene transcripts in the other phenotypes of plant. Data shown are mean values \pm SD bars of results from three experimental repeats (15 plants/repeat). Different letters on bar graphs indicate significant $(P<0.01)$ differences by one-way ANOVA and LSD test.

the transcript abundance of a relatively small number of $A R F$ gene candidates and the effect is either positive or negative depending on particular candidates.

\section{Discussion}

The repressive effect of the NtTTG2 gene silencing and the promoting effect of the gene overexpression on vegetative growth, floral anthocyanin synthesis, flower colorization, and seed production (Figures 1, 2 and 3; Additional file 1: Figure S1, Additional file 2: Figures S2) suggest that NtTTG2 is an essential regulator of the growth and development processes in tobacco N. tabacum. The growth/developmental role of NtTTG2 associates with its effects on the expression of EXP, DFR, and ANS genes, which are essentially involved in the plant growth and development pathways $[6,48,57,58]$.

EXPs have unique "loosening" effects on plant cell walls [58] and promote cell expansion [58-61] and plant growth $[60,62,63]$ under regulation by phytohormones including auxin $[64,65]$. Of six EXP genes identified in tobacco and hundreds of EXPs in other plants ([57,58,66,67]; http://www.personal.psu.edu/fsl/ ExpCentral]), EXP1 and EXP2 are widely characterized to be required for plant growth [57,58,62-64]. EXP1 and EXP2 expression consistent with the biomass (Figures 1 and 3) suggests that both EXPs contribute to NtTTG2mediated vegetative growth of tobacco. Since the expression of EXPs is subject to multiple regulations [64,65], it is not surprising that NtTTG2 silencing reduces but does not eliminate EXP expression.

NtTTG2 differently affects the expression of genes involved in the anthocyanin biosynthesis pathway (Figures 2 and 3; Additional file 2: Figure S2). NtTTG2 promotes the expression of the DFR and ANS genes, which function at the penultimate and antepenultimate steps, respectively in the anthocyanin biosynthesis pathway that mainly involves 
nine genes [6,50]. DFR encodes dihydroflavonol 4-reductase that reduces three dihydroflavonols to three leucoanthocyanidins $[6,60,68]$, and then two leucoanthocyanidins are converted by ANS-encoded anthocyanin synthase to anthocyanidin [69]. The other seven NtTTG2-independent genes (Additional file 2: Figure S2) encode enzymes with specific actions on single substrates $[6,50]$. Therefore, NtTTG2 affects anthocyanin production and flower colorization through affecting the expression of genes critical to the final formation of anthocyanin (Figures 2 and 3; Additional file 2: Figure S2). In addition, severe abortions owing to NtTTG2 silencing and marked promotion of the seed production by the gene overexpression suggest that $N t T T G 2$ plays a role in seed development (Figures 3 and 5). How NtTTG2 regulates the expression of DFR and $A N S$, and EXPs as well, remains to be studied.

To reveal the molecular mechanism that underlies the growth/developmental role of NtTTG2, we performed de novo assembly of the tobacco $N$. tabacum transcriptome to identify NtTTG2-affected ARF gene candidates in the case that little were known about the regulation of auxin responses in tobacco $[40,41,43]$. The transcriptome was assembled with a high quality based on proportion of clean reads in total raw reads and length sorting of unigenes (Figure 4). High quality of the transcriptome assembling can be also found in significant homology of unigene sequences with those searched from the public databases (Figures 5 and 6). Analyses of the transcriptome have created substantial data on functional components in the plant genome: a total of 54,906 unigenes, 30,124 unigenes (54.86\%) with annotated functions, at least 8,024 unigenes (14.61\%) assigned to plant growth and development, and 455 unigenes (0.83\%) related to the regulation of auxin responses (Figures 5, 7, 8 and 9) including 40 putative $A R F$ genes (Figure 10; Additional file 3: Table S1). In addition, 24,782 (45.14\%) unigenes do not have annotation in any of the databases.

With such substantial genetic components, the $N$. tabacum transcriptomics inventory should be useful in further studies on a broad perspective of the plant genetics and on physiological or biochemical connections between TTGs and auxin responses in particular. In support of this notion, we show that NtTTG2 significantly affects transcript abundances of 13 of 40 putative $A R F$ genes and the effect is either positive or negative depending on particular unigenes (Figure 11). This indicates that NtTTG2 may function in association with ARF transcription factors in regulating the plant growth and development. In a recently published draft genome sequence of tobacco $N$. benthamiana (http://sol genomics.net/organism/Nicotiana_benthamiana/genome), 24 immunity-associated genes were retrieved from approximately 16,000 unigenes and this was regarded as evidence for the usefulness of the genome assembly in the study of plant-pathogen interactions [70]. In comparison, the effects of NtTTG2 on transcript abundances of putative $A R F$ genes (Figure 11) suggest that the $N$. tabacum transcriptome is useful to the study of auxin responses in the plant.

However, the current technique used in de novo transcriptome assembling is unable to obtain full-length sequences of most unigenes. Instead, most unigenes are identified based on partial sequences present in the transcriptome. In this regard, it is necessary to perform a comprehensive comparison between the 54,906 unigenes identified in the $N$. tabacum transcriptome and informative genomic sequence of the plant. The $N$. benthamiana genome sequence was established by assembling of 461,463 sequences with an average length of 5,336 bp [70]. In the current study, the N. tabacum transcriptome was assembled from 111,207 transcripts with an average length of 1,031 bp. Thus, it is rational to speculate that unigenes identified in this study must be much shorter than the actual length of real genes. Due to this reason, further characterizing these unigenes is a great challenge.

It is also a great challenge to characterize the functional relationship between NtTTG2 and ARFs in tobacco. Full-length sequences of the putative $A R F$ genes remain unknown except for comp42904_c0, which is $100 \%$ identical with the sequence of the $A R F 8$ gene from the $N$. tabacum variety NC89 (JN835423.1). The predicted ARF8 protein is characteristic of transcription activators based on glutamine abundance in the middle region of the protein sequence, in contrast to serine abundance in the same region of an ARF transcription repressor [25]. Unfortunately, information available from the $N$. tabacum transcriptome is insufficient to show whether the $39 A R F$ candidates encode transcription activators or repressors. Cloning of full-length sequences of the ARF candidates, analyses of their roles as transcription activators or repressors, and characterization of their functional relationships with NtTTG2 will be the subject of further studies.

\section{Conclusions}

Molecular and genetic analyses performed in this study demonstrate that NtTTG2 plays an indispensable role in the regulation of growth and development processes in tobacco $N$. tabacum. The growth/developmental role of NtTTG2 associates with its function in regulating the expression of growth-promoting EXP genes as well as the DFR and ANS genes essentially involved in the anthocyanin biosynthesis pathway. In the growth/ developmental regulation, moreover, NtTTG2 may have a functional linkage with auxin responses as indicated by the effects of NtTTG2 silencing and overexpression on the expression of 13 putative $A R F$ genes identified in the tobacco transcriptome assembled by the de novo assembly 
method. Substantial data of genetic components derived from analyses of the transcriptome should be useful for studies in the future on the plant genetics in general and on the functional relationship between NtTTG2 and auxin in regulating growth and development of the plant in particular.

\section{Methods}

\section{Plant material and growth conditions}

The WT and transgenic plants of the tobacco ( $N$. tabacum) variety NC89 were used in this study. Transgenic plants included the NtTTG2-silencing line TTG2RNAi4, NtTTG2-overexpressing P35S:TTG2:RFP lines, and transgenic control line WT RFP generated previously [5]. Seedlings used in monitoring of root growth were grown on MS agar medium in 10-cm square plates incubated in an environment-controlled chamber with a 14-hour-light $\left(250 \mu \mathrm{E} / \mathrm{m}^{2} / \mathrm{s}\right.$ at $\left.26^{\circ} \mathrm{C}\right)$ and 10 -hour-dark $\left(23^{\circ} \mathrm{C}\right)$ cycle. Plants used in developmental observations were grown a mixture of sand and potting soil in $360-\mathrm{mL}$ pots incubated in a greenhouse at $23-26^{\circ} \mathrm{C}$.

\section{Plant growth, development, and anthocyanin analyses}

The vegetative growth and development of flowers and seeds were monitored. Total anthocyanin was extracted by homogenizing fresh flowers in liquid nitrogen. Fine flower powders were immediately lyophilized and maintained at $-80^{\circ} \mathrm{C}$ until use. Total anthocyanin in lyophilized flower powders was extracted by incubation in methanol solution containing $1 \%$ hydrochloric acid for $18 \mathrm{~h}$ at room temperature and under moderate shaking. The extract suspension was centrifuged $\left(12,000 \mathrm{~g}, 4^{\circ} \mathrm{C}\right.$, $1 \mathrm{~min}$ ) to precipitate cellular debris and collect supernatant. Anthocyanin concentration in the supernatant was quantified by spectrophotometry and the endogenous content was scored in contrast to fresh weight of flowers used in the extract preparation.

\section{RNA isolation and quality verification}

Total RNA used in gene expression analysis was isolated from the fourth youngest leaves of 40-day-old plants grown in ports and S1-S5 flowers or 15-day-old immature fruits (capsules) of proper plants grown in pots. Total RNA used in the transcriptome assembly was isolated from the fourth youngest leaves of 40-day-old plants grown in pots. In order to eliminate the variation between individual plants, three biological repetitions were performed and in each repetition, RNA was isolated from a mixture of five leaves each from an individual plant. The collected leaves were homogenized in liquid nitrogen. RNA was isolated from frozen leaf powders were performed by using TRIzolH Reagent (Invitrogen, San Diego, USA) and then treated with DNase I (Invitrogen). The quality of RNA samples was verified using a 2100
Bioana-lyzer RNA Nanochip (Agilent, Santa Clara, CA) with the criteria of RIN (RNA Integrity Number) greater than 8.5 and $28 \mathrm{~S}: 18 \mathrm{~S}$ greater than 1.5. Concentrations of aqueous RNA preparations were determined by spectrophotometry at the standard of $\mathrm{OD}_{260 / 280}$ between 1.8 and 2.2 along with $\mathrm{OD}_{260 / 230}$ greater than 1.8.

\section{Gene expression analysis}

Gene expression was determined by real-time reverse transcriptase-polymerase chain reaction (RT-PCR) using specific primers (Additional file 4: Tables S2 and Additional file 5: Table S3) and previously described protocols [71,72]. The constitutively expressed $E F 1 \alpha$ gene was used as a reference [62,63,71-73]. The expression level of a tested gene was quantified as the transcript ratio to $E F 1 \alpha$.

\section{cDNA library construction and sequencing}

Equal quantities of total RNA from three samples were mixed to prepare the pooled RNA sample for RNA-Seq. A total amount of $30 \mu \mathrm{g}$ mixed RNA sample confirmed for RIN values above 8.0 was used as input material in constructing the sequencing library. The library was generated using Illumina TruSeq ${ }^{\text {tin }}$ RNA Sample Preparation Kit (Illumia, San Diego, USA) as per the manufacturer's recommendations and a tetrad index code was added to the sample for subsequent documentation. High quality mRNA was obtained by purification of the total RNA sample using poly- $T$ oligo-attached magnetic beads. Fragmentation was carried out using divalent cations under elevated temperature in Illumina proprietary fragmentation buffer. First strand cDNA was synthesized using random oligonucleotides and SuperScript II. Second strand cDNA synthesis was subsequently performed using DNA polymerase I and RNase $\mathrm{H}$. Remaining overhangs were converted into blunt ends via exonuclease/ polymerase activities and enzymes were removed. After adenylation of 3'-terminal ends of DNA fragments, Illumina PE adapter oligonucleotides were ligated to prepare for hybridization. In order to select cDNA fragments of preferentially $200 \mathrm{bp}$ in length, the library fragments were purified with AMPure XP system (Beckman Coulter, Beverly, USA). DNA fragments with ligated adaptor molecules on both ends were selectively enriched using Illumina PCR Primer Cocktail in a 10 cycle PCR reaction. Products were purified (AMPure XP system) and quantified using the Agilent high sensitivity DNA assay on the Agilent Bioanalyzer 2100 system. The clustering of the index-coded sample was performed on a cBot Cluster Generation System using TruSeq PE Cluster Kit v3-cBot-HS (Illumia) according to the vender's instructions. After cluster generation, the library preparation was sequenced on an Illumina Hiseq 2000 platform and 100 bp pairedend reads were generated. 


\section{Data filtering and de novo assembly}

Image data output from the sequencing device were transformed into raw reads and stored in FASTQ format. Clean reads were obtained after removing reads that contained adaptor sequences, reads in which more than $10 \%$ of the bases were unknown, and reads in which more than half of the quality values of the bases were less than 5. De novo assembly was carried out using Trinity [74]. Resulting unigenes were analyzed by searching the Nr, Nt, Pfam, KOG/COG, Swiss-Prot, KEGG, and $\mathrm{GO}$ databases. If results obtained using different databases conflicted with each other, the sequence direction of unigenes was determined by a priority order of NR, Swiss-Prot, KEGG, and KOG. Information obtained from BLAST was used to extract CDS from unigene sequences and translate them into peptide sequences. Unigenes with no hits in BLAST were analyzed with ESTScan [75] to predict their coding regions and determine their sequence direction.

\section{Gene annotation and function classification}

Unigenes were annotated and proteins were predicted using the nucleotide $(\mathrm{Nt})$ and protein $(\mathrm{Nr}, \mathrm{Pfam}$, and Swiss-Prot) databases and assigned to functional categories in the KOG/COG, GO, and KEGG databases by BLASTx searches with E value cutoff of $10^{-5}$. With $\mathrm{Nr}$ annotation, unigenes were assigned by the Blast $2 \mathrm{GO}$ program $[53,76]$ to molecular function, biological process and cellular component ontologies of the GO database (http:// www.Geneontology.org). Unigenes were also searched against the KOG/COG database to predict and classify possible functions. Pathway assignments were carried out according to the Kyoto Encyclopedia of Genes and Genomes pathway database [56] also using BLASTx with $E$ value threshold of $10^{-5}$.

\section{Data analysis}

All experiments were carried out at least three times with similar results. Quantitative data were analyzed by ANOVA and Fisher's Least Significant Difference (LSD) test.

\section{Availability of supporting data}

The tobacco transcriptome data are available at http:// www.ncbi.nlm.nih.gov/sra/?term=SRX363387.

\section{Additional files}

Additional file 1: Figure S1. Comparison of the wild-type (WT) tobacco plant and the transgenic control line WT RFP in terms of growth and development processes.

Additional file 2: Figure S2. Real-time RT-PCR analyses of tobacco genes involved in the anthocyanin biosynthesis pathway and expressed in flowers at developmental stages S1-S5.

Additional file 3: Table S1. Genes identified in the tobacco transcriptome and annotated as putative ARF genes by searches against seven databases.
Additional file 4: Table S2. Information on real-time RT-PCR analyses of putative ARF genes identified in the tobacco transcriptome.

Additional file 5: Table S3. Information on genes identified previously and primers used in this study.

\section{Abbreviations}

ARF: AUXIN RESPONSE FACTOR; BLAST: Basic local alignment search tool; EST: Expressed sequence tag; GO: Gene ontology; KEGG: Kyoto encyclopedia of genes and genomes database; KOG/COG: EuKaryotic ortholog groups/ clusters of orthologous groups of proteins; Nr: NCBI Non-redundant protein sequences; Nt: NCBI Nucleotide sequences; Pfam: Protein family; P35S:TTG2: RFP: The transgenic plant overexpressing the NtTTG2:RFP fusion gene under control by the cauliflower mosaic virus 355 promoter; RT-PCR: Reverse transcriptase-polymerase chain reaction; TTG: TRANSPARENT TESTA GLABRA; TTG2RNAi: The NtTTG2 silencing transgenic plant; WT RFP: The transgenic plant expressing the RFP gene coding for red-fluorescent protein.

\section{Competing interests}

The authors have declared that no competing interests exist.

\section{Authors' contributions}

$\mathrm{QZ}$ and BL designed and performed the experiments and wrote the paper. SM performed the experiments, analyzed the data, and wrote the paper. $\mathrm{BH}$, $Z C$, and ZY performed the experiments. MX edited the manuscript. HD designed the experiments and wrote the paper. All authors read and approved the final manuscript.

\section{Acknowledgements}

This study was supported by NSFC (31171830 and 31272072), National Key Basic Research Program of China (973 plan 2012CB114003), Novel Transgenic Organisms Breeding Project (2011ZX08002-001), and Ministry of Education 111 Project of China and Academic Priority Program of High Education in Jiangsu Province.

\section{Author details}

${ }^{1}$ Plant Growth and Defense Signaling Laboratory, State Ministry of Education Key Laboratory of Integrated Management of Crop Pathogens and Insect Pests, Nanjing Agricultural University, Nanjing 210095, China. ${ }^{2}$ Yantai Academy of Agricultural Sciences, Yantai 265500, China.

Received: 19 July 2013 Accepted: 12 November 2013

Published: 20 November 2013

\section{References}

1. Bouyer D, Geier F, Kragler F, Schnittger A, Pesch M, Wester K, Balkunde R, Timmer J, Fleck C, Hülskamp M: Two-dimensional patterning by a trapping/depletion mechanism: the role of $\Pi \mathrm{TG} 1$ and GL3 in Arabidopsis trichome formation. PLOS Biol 2008, 6:1166-1177.

2. Petroni $\mathrm{K}$, Tonelli $\mathrm{C}$ : Recent advances on the regulation of anthocyanin synthesis in reproductive organs. Plant Sci 2011, 181:219-229.

3. Szymanski DB, Lloyd AM, Marks MD: Progress in the molecular genetic analysis of trichome initiation and morphogenesis in Arabidopsis. Trends Plant Sci 2000, 5:214-219.

4. Vetten N, Quattrocchio F, Mol J, Koes R: The an11 locus controlling flower pigmentation in petunia encodes a novel WD-repeat protein conserved in yeast, plants and animals. Gene Dev 1997, 11:1422-1434.

5. Li B, Gao R, Cui R, Lü B, Li X, You Z, Dong H: Tobacco TTG2 suppresses resistance to pathogens by sequestering NPR1 from the nucleus. J Cell Sci 2012, 125:4913-4922.

6. Pang $Y$, Wenger JP, Saathoff K, Peel GJ, Wen J, Huhman D, Allen SN, Tang Y, Cheng $X$, Tadege M, Ratet P, Mysore KS, Sumner LW, Marks MD, Dixon RA: A WD40 repeat protein from Medicago truncatula is necessary for tissuespecific anthocyanin and proanthocyanidin biosynthesis but not for trichome development. Plant Physiol 2009, 151:1114-1129.

7. Wang Y, Liu R, Chen L, Wang YC, Liang YC, Wu X, Li B, Wu J, Liang Y, Wang X, Zhang C, Wang Q, Hong X, Dong H: Nicotiana tabacum TTG1 contributes to ParA1-induced signalling and cell death in leaf trichomes. J Cell Sci 2009, 122:2673-2685.

8. Neer EJ, Schmidt CJ, Nambudripad R, Smith TF: The ancient regulatoryprotein family of WD-repeat proteins. Nature 1994, 371:297-300. 
9. Biedermann S, Hellmann H: WD40 and CUL4-based E3 ligases: lubricating all aspects of life. Trends Plant Sci 2011, 16:38-46.

10. Stirnimann CU, Petsalaki E, Russell RB, Müller CW: WD40 proteins propel cellular networks. Trends Biochem Sci 2010, 35:565-574.

11. Xu C, Min J: Structure and function of WD40 domain proteins. Protein Cell 2011, 2:202-214

12. Johnson CS, Kolevski B, Smyth DR: TRANSPARENT TESTA GLABRA2, a trichome and seed coat development gene of Arabidopsis, encodes a WRKY transcription factor. Plant Cell 2002, 14:1359-1375.

13. Morohashi K, Zhao M, Yang M, Read B, Lloyd A, Lamb R, Grotewold E: Participation of the Arabidopsis bHLH factor GL3 in trichome initiation regulatory events. Plant Physiol 2007, 145:736-746.

14. Ghanashyam C, Jain M: Role of auxin-responsive genes in biotic stress responses. Plant Signal Behav 2009, 4:846-848.

15. Truman WM, Bennett MH, Turnbull CGN, Grant MR: Arabidopsis auxin mutants are compromised in systemic acquired resistance and exhibit aberrant accumulation of various indolic compounds. Plant Physiol 2010, 152:1562-1573.

16. Walker AR, Davison PA, Bolognesi-Winfield AC, James $C M$, Srinivasan $N$, Blundell TL, Esch JJ, Marks MD, Gray JC: The TRANSPARENT TESTA GLABRA1 locus, which regulates trichome differentiation and anthocyanin biosynthesis in Arabidopsis, encodes a WD40 repeat protein. Plant Cell 1999, 11:1337-1350.

17. Zhao M, Morohashi K, Hatlestad G, Grotewold E, Lloyd A: The TTG1-bHLHMYB complex controls trichome cell fate and patterning through direct targeting of regulatory loci. Development 2008, 135:1991-1999.

18. Kamoun S, Klucher KM, Coffey MD, Tyler BM: A gene encoding a hostspecific elicitor protein of Phytophthora parasitica. Mol Plant Microbe Interact 1993, 6:15-25.

19. Wang YC, Li B, Wang Y, Dong H: Cloning of full-length NtTTG1-like CDNA in Nicotiana tabacum, and the analysis of its expression and subcellular localization. Jiangsu Agric Sci 2010, 1:23-26.

20. Cao H, Glazebrook J, Clarke JD, Volko S, Dong X: The Arabidopsis NPR 1 gene that controls systemic acquired resistance encodes a novel protein containing ankyrin repeats. Cell 1997, 88:57-63.

21. Mukhtar MS, Nishimura MT, Dangl J: NPR1 in plant defense: It's not over 'til it's turned over. Cell 2009, 137:805-806.

22. Spoel SH, Mou Z, Tada Y, Spivey NW, Genschik P, Dong X: Proteasomemediated turnover of the transcription coactivator NPR1 plays dual roles in regulating plant immunity. Cell 2009, 137:860-872.

23. Quint M, Gray WG: Auxin signaling. Curr Opin Plant Biol 2006, 9:448-453.

24. Vanneste S, Friml J: Auxin: A trigger for change in plant development. Cell 2009, 136:1005-1016

25. Guilfoyle TJ, Ulmasov T, Hagen G: The ARF family of transcription factors and their role in plant hormone-responsive transcription. Cell Mol Life Sci 1998, 54:619-627.

26. Kumar R, Agarwal P, Tyagi AK, Sharma AK: Genome-wide investigation and expression analysis suggest diverse roles of auxin-responsive $G H 3$ genes during development and response to different stimuli in tomato (Solanum lycopersicum). Mol Genet Genomics 2012, 287:221-235.

27. Liscum E, Reed JW: Genetics of Aux/IAA and ARF action in plant growth and development. Plant Mol Biol 2002, 49:387-400.

28. Walcher CL, Nemhauser JL: Bipartite promoter element required for auxin response. Plant Physiol 2012, 158:273-282

29. Tiwari SB, Hagen G, Guilfoyle T: The roles of auxin response factor domains in auxin-responsive transcription. Plant Cell 2003, 15:533-543.

30. Ulmasov T, Hagen G, Guilfoyle TJ: Activation and repression of transcription by auxin-response factors. Proc Natl Acad Sci USA 1999, 96:5844-5849

31. Guilfoyle TJ, Hagen G: Auxin response factors. Curr Opin Plant Biol 2007, 10:453-460.

32. Hagen G, Guilfoyle T: Auxin-responsive gene expression: genes, promoters and regulatory factors. Plant Mol Biol 2002, 49:373-385.

33. Banks JA, Nishiyama T, Hasebe M, Bowman JL, Gribskov M, dePamphilis C, Albert VA, Aono N, Aoyama T, Ambrose BA, Ashton NW, Axtell MJ, Barker E, Barker MS, Bennetzen JL, Bonawitz ND, Chapple C, Cheng C, Correa LG, Dacre M, DeBarry J, Dreyer I, Elias M, Engstrom EM, Estelle M, Feng L, Finet C, Floyd SK, Frommer WB, Fujita T, et al: The Selaginella genome identifies genetic changes associated with the evolution of vascular plants. Science 2011, 332:960-963.

34. Ha CV, Le DT, Nishiyama R, Watanabe Y, Sulieman S, Tran UT, Mochida K, Dong NV, Yamaguchi-Shinozaki K, Shinozaki K, Tran LS: The auxin response factor transcription factor family in soybean: Genome-wide identification and expression analyses during development and water stress. DNA Res 2013. doi:10.1093/dnares/dst027.

35. Kalluri UC, Difazio SP, Brunner AM, Tuskan GA: Genome-wide analysis of Aux/ IAA and ARF gene families in Populus trichocarpa. BMC Plant Biol 2007, 7:59.

36. Rensing SA, Lang D, Zimmer AD, Terry A, Salamov A, Shapiro H, Nishiyama T, Perroud PF, Lindquist EA, Kamisugi Y, Tanahashi T, Sakakibara K, Fujita T, Oishi K, Shin-I T, Kuroki Y, Toyoda A, Suzuki Y, Hashimoto S, Yamaguchi K, Sugano S, Kohara Y, Fujiyama A, Anterola A, Aoki S, Ashton N, Barbazuk WB, Barker E, Bennetzen JL, Blankenship R, et al: The Physcomitrella genome reveals evolutionary insights into the conquest of land by plants. Science 2008, 319:64-69.

37. Tromas A, Perrot-Rechenmann C: Recent progress in auxin biology. CR Biol 2010, 333:297-306.

38. Heinekamp T, Strathmann A, Kuhlmann M, Froissard M, Müller A, Catherine $P R$, Wolfgang DL: The tobacco bZIP transcription factor BZI-1 binds the GH3 promoter in vivo and modulates auxin-induced transcription. Plant J 2004, 38:298-309.

39. Liu ZB, Ulmasov T, Shi X, Hagen G, Guilfoyle TJ: The soybean GH3 promoter contains multiple auxin-inducible elements. Plant Cell 1994, 6:645-657.

40. Ulmasov T, Liu ZB, Hagen G, Guilfoyle TJ: Composite structure of auxin response elements. Plant Cell 1995, 7:1611-1623.

41. Wu J, Liu SY, He YJ, Guan XY, Zhu XF, Cheng L, Wang J, Lu G: Genome-wide analysis of SAUR gene family in Solanaceae species. Gene 2012, 509:38-50.

42. Yuan HZ, Zhao K, Lei HJ, Shen XJ, Liu Y, Liao X, Li TH: Genome-wide analysis of the GH3 family in apple (Malus $\times$ domestica). BMC Genomics 2013, 14:297. doi:10.1186/1471-2164-14-297.

43. Todd AT, Liu E, Polvi SL, Pammett RT, Page JE: A functional genomics screen identifies diverse transcription factors that regulate alkaloid biosynthesis in Nicotiana benthamiana. Plant J 2010, 62:589-600.

44. Kumar R, Tyagi AK, Sharma AK: Genome-wide analysis of auxin response factor (ARF) gene family from tomato and analysis of their role in flower and fruit development. Mol Genet Genomics 2011, 285:245-260.

45. Sanchez MA, Mateos I, Labrador E, Dopico B: Brassinolides and IAA induce the transcription of four a-expansin genes related to development in Cicer arietinum. Plant Physiol Biochem 2004, 42:709-716.

46. Yi L, Jones L, McQueen-Mason S: Expansins and cell growth. Curr Opin Plant Biol 2003, 6:603-610.

47. Asen S, Stewart RN, Norris KH: Co-pigmentation of anthocyanins in plant tissues and its effect on color. Phytochemistry 1972, 11:1139-114.

48. Hichri I, Barrieu F, Bogs J, Kappel C, Delrot S, Lauvergeat V: Recent advances in the transcriptional regulation of the flavonoid biosynthetic pathway. J Exp Bot 2011, 62:2465-2483.

49. Saito $K$, Yamazaki M: Biochemistry and molecular biology of the latestage of biosynthesis of anthocyanin: lessons from Perilla frutescens as a model plant. New Phytol 2002, 155:9-23.

50. Tanaka Y, Brugliera F, Kalc G, Senior M, Dyson B, Nakamura N, Katsumoto Y, Chandler S: Flower color modification by engineering of the flavonoid biosynthetic pathway: practical perspectives. Biosci Biotech Biochem 2010 74:1760-1769

51. Góngora-Castillo E, Buell CR: Bioinformatics challenges in de novo transcriptome assembly using short read sequences in the absence of a reference genome sequence. Nat Prod Rep 2013, 30:490-500.

52. Van Belleghem SM, Roelofs D, Van Houdt J, Hendrickx F: De novo transcriptome assembly and SNP discovery in the wing polymorphic salt marsh beetle Pogonus chalceus (Coleoptera, Carabidae). PLoS One 2012, 7:e42605.

53. Götz S, García-Gómez JM, Terol J, Williams TD, Nagaraj SH, Nueda SH, Robles M, Talón M, Dopazo J, Conesa A: High-throughput functional annotation and data mining with the Blast2GO suite. Nucleic Acids Res 2008, 36:3420-3435.

54. Ye J, Fang L, Zheng H, Zhang Y, Chen J, Zhang Z, Wang J, Li S, Li R, Bolund L: WEGO: a web tool for plotting GO annotations. Nucleic Acids Res. 2006, 34:W293-297.

55. Moon EK, Chung DI, Hong YC, Ahn Tl, Kong HH: Acanthamoeba castellanii: Gene profile of encystation by ESTs analysis and KOG assignment. Exp Parasitol 2008, 119:111-116.

56. Kanehisa M, Goto S: KEGG: Kyoto encyclopedia of genes and genomes. Nucleic Acids Res 2000, 28:27-30.

57. Sloan J, Backhaus A, Malinowski R, McQueen-Mason S, Fleming AJ: Phased control of expansin activity during leaf development identifies a sensitivity window for expansin-mediated induction of leaf growth. Plant Physiol 2009, 151:1844-1854. 
58. Wang G, Gao Y, Wang J, Yang L, Song R, Li X, Shi J: Overexpression of two cambium-abundant Chinese fir (Cunninghamia lanceolata) a-expansin genes CIEXPA1 and CIEXPA2 affect growth and development in transgenic tobacco and increase the amount of cellulose in stem cell walls. Plant Biotechnol J 2011, 9:486-502.

59. Cosgrove DJ: Loosening of plant cell walls by expansins. Nature 2000, 407:321-326.

60. Lee $\mathrm{Y}$, Kende $\mathrm{H}$ : Expression of $\beta$-expansins is correlated with internodal elongation in deepwater rice. Plant Physiol 2001, 127:645-654.

61. Nieuwland J, Feron R, Huisman BA, Fasolino A, Hilbers CW, Derksena J, Mariani C: Lipid transfer proteins enhance cell wall extension in tobacco. Plant Cell 2005, 17:2009-2019.

62. Liu F, Liu H, Jia Q, Wu X, Guo X, Guo X, Zhang S, Song F, Dong H: The internal glycinea-rich motif and cysteine suppress several effects of the $\mathrm{HpaG}_{\text {xooc }}$ protein in plants. Phytopathology 2006, 10:1052-1059.

63. Ren H, Gu G, Long J, Yin Q, Wu T, Song T, Zhang S, Chen Z, Dong H: Combinative effects of a bacterial type-III effector and a biocontrol bacterium on rice growth and disease resistance. J Biosci 2006, 31:617-627.

64. Catala C, Rose JK, Bennett AB: Auxin-regulated genes encoding cell wallmodifying proteins are expressed during early tomato fruit growth. Plant Physiol 2000, 122:527-534.

65. Cox MC, Benschop JJ, Vreeburg RA, Wagemaker CA, Moritz T, Peeters AJ, Voesenek $L A$ : The roles of ethylene, auxin, abscisic acid, and gibberellin in the hyponastic growth of submerged Rumex palustris petioles. Plant Physiol 2004, 136:2948-2960.

66. Breuer C, Ishida T, Sugimoto K: Developmental control of endocycles and cell growth in plants. Curr Opin Plant Biol 2010, 13:654-660.

67. Li F, Xing S, Guo Q, Zhao M, Zhang J, Gao Q, Wang G, Wang W: Drought tolerance through over-expression of the expansin gene TaEXPB23 in transgenic tobacco. J Plant Physiol 2011, 168:960-966.

68. Goldsbrough A, Belzile F, Yoder J: Complementation of the tomato anthocyanin without (aw) mutant using the dihydroflavonol 4-reductase gene. Plant Physiol 1994, 105:491-496.

69. Xie D, Sharma SB, Paiva NL, Ferreira D, Dixon RA: Role of anthocyanidin reductase, encoded by BANYULS in plant flavonoid biosynthesis. Science 2003, 299:396-399.

70. Bombarely A, Rosli HG, Vrebalov J, Moffett P, Mueller LA, Martin GB: A draft genome sequence of Nicotiana benthamiana to enhance molecular plantmicrobe biology research. Mol Plant Microbe Interact 2012, 25:1523-1530.

71. Chen L, Qian J, Qu S, Long J, Yin Q, Zhang C, Wu X, Sun F, Wu T, Beer SV, Dong $\mathrm{H}$ : Identification of specific fragments of $\mathrm{HpaG}_{\mathrm{Xooc}_{\mathrm{o}}}$ a harpin protein from Xanthomonas oryzae pv. oryzicola, that induce disease resistance and enhanced growth in rice. Phytopathology 2008, 98:781-791.

72. Liu R, Chen L, Jia Z, Lü B, Dong H: Transcription factor AtMYB44 regulates induced expression of the ETHYLENE INSENSITIVE2 gene in Arabidopsis responding to a harpin protein. Mol Plant Microbe Interact 2010, 24:377-389.

73. Peng J, Bao Z, Ren H, Wang J, Dong H: Expression of harpin Xoo $_{\text {in }}$ transgenic tobacco induces pathogen defense in the absence of hypersensitive response. Phytopathology 2004, 94:1048-1055.

74. Grabherr MG, Haas BJ, Yassour M, Levin JZ, Thompson DA, Amit I, Adiconis X, Fan L, Raychowdhury R, Zeng Q, Chen Z, Mauceli E, Hacohen N, Gnirke A, Rhind N, di Palma F, Birren BW, Nusbaum C, Lindblad-Toh K, Friedman N, Regev A: Full-length transcriptome assembly from RNA-Seq data without a reference genome. Nat Biotechnol 2011, 29:644-652.

75. Kim H, Schmidt CJ, Decker KS, Emara MG: A double-screening method to identify reliable candidate non-synonymous SNPs from chicken EST data. Anim Genet 2003, 34:249-54.

76. Conesa A, Gotz S, Garcia-Gomez JM, Terol J, Talon M, Robles M: Blast2GO: a universal tool for annotation, visualization and analysis in functional genomics research. Bioinformatics 2005, 21:3674-3676.

doi:10.1186/1471-2164-14-806

Cite this article as: Zhu et al.: TTG2-regulated development is related to expression of putative AUXIN RESPONSE FACTOR genes in tobacco. BMC Genomics 2013 14:806.

\section{Submit your next manuscript to BioMed Central and take full advantage of:}

- Convenient online submission

- Thorough peer review

- No space constraints or color figure charges

- Immediate publication on acceptance

- Inclusion in PubMed, CAS, Scopus and Google Scholar

- Research which is freely available for redistribution

Submit your manuscript at www.biomedcentral.com/submit
C BioMed Central 